\title{
La competencia depurar sistemas informáticos en la Carrera de Ingeniería Informática de la Universidad de Matanzas
}

\author{
Walfredo González Hernández. Universidad de Matanzas \\ Recepción: 22.03.2019 | Aceptado: 15.10.2019 \\ Correspondencia a través de ORCID: Walfredo González Hernández \\ iD 0000-0001-8974-3721 \\ Citar: Gonzalez, W. (2019). La depuración como competencia en la formación del profesional informático. \\ ReiDoCrea, 8, 119-132.
}

\begin{abstract}
Resumen: La depuración es una de las actividades más importantes en la actividad ingenieril. En el artículo se toma posiciones de los autores sobre el modelo y sus clasificaciones. Por otro lado, se asumen los elementos esenciales del currículo informático en el mundo y la importancia de la depuración en ellos. En el último acápite se analiza el cómo estructurar la depuración como elemento esencial en su modo de actuación. Se parametriza la variable de estudio y de determinan sus dimensiones e indicadores. Posteriormente se analiza la evolución de la variable a partir de la introducción de la asignatura gestión de pruebas y la realización de un proyecto real.
\end{abstract}

Palabras clave: Enseñanza de la Informática | Competencias

The competence debug computer systems in the Computer Engineering Career of the University of Matanzas

Abstract: Debugging is one of the most important activities in engineering activity. In the article, the author's positions on the model and its classifications are taken. On the other hand, they assume the essential elements of the computer curriculum in the world and the importance of the debugging in them. The last section discusses how to structure debugging as an essential element in its mode of action. The study variable is parameterized and its dimensions and indicators are determined. Subsequently we analyze the evolution of the variable from the introduction of the subject test management and the realization of a real project.

Keywords: Informatics teach | Competence

\section{Introducción}

El desarrollo alcanzado por las Tecnologías de la Información y la Comunicación (TIC) y sus aplicaciones en la educación a nivel mundial y en el ámbito nacional, plantean la necesidad de investigar un conjunto de problemas inherentes al proceso de enseñanzaaprendizaje de la Informática. Uno de estos problemas es la formación del profesional de esta área del conocimiento.

La informática ha desarrollado en su devenir histórico varias disciplinas científicas que se estructuran como disciplinas en el currículo de su formación. Sin embargo, existen determinados contenidos que son esenciales para su formación y que no se encuentran enmarcados en una única disciplina. Uno de estos contenidos es precisamente la modelación.

La depuración de los sistemas informáticos es una de las actividades más importantes en la formación del ingeniero informático. Sin embargo, el análisis debe estar en el orden de las estructuras psicológicas de la personalidad que se debe considerar como depuración de los sistemas informáticos en la formación del profesional informático. Un primer punto de partida en este análisis es el papel de la depuración en la actividad informática y su forma fundamental de organización. Un segundo momento de análisis se encuentra en la expresión de la depuración en el diseño curricular de los 
profesionales informáticos. Por último, entonces determinar qué tipo de estructura de la personalidad ocupa la depuración en la formación de este profesional.

Los procesos de informatización de las organizaciones transcurren desde el análisis de sus necesidades hasta que éstas se satisfacen. Durante todos estos procesos se van construyendo diferentes modelos y éstos se van concretando en la medida del proceso de informatización que se realice. Estos procesos de informatización transcurren en forma de proyectos, lo cual se asume como la forma fundamental de organización de la actividad informática.

Asumir que el proyecto es la forma fundamental de la organización de la actividad informática implica reconocer que es un proceso colaborativo en el cual intervienen un conjunto de personas. También que estas personas tienen determinadas labores que desempeñan en este proceso a las cuales se les denomina roles (Díaz, 2015; Pressman, 2010) aunque algunas de ellas pueden ocupar más de uno. También se reconoce que el desarrollarlo como proyecto implica un sistema de acciones con carácter de sistema que lleve a la culminación del proceso. Este sistema de acciones generalmente comienza con las especificaciones del cliente, posteriormente continúa con la modelación de los procesos de informatización, la implementación de los modelos y seguidamente la depuración del proceso que se ha realizado. Se trata de depurar los errores cometidos durante los procesos que antecedieron en la práctica, esta vez no es solamente para comprobar la veracidad del modelo obtenido sino además para completar una fase de concreción del proceso de informatización. Ejes transversales a estos procesos lo constituyen las depuraciones de los errores cometidos y el aseguramiento de la calidad de estos procesos de tal manera que se realice un proceso con calidad.

Siguiendo estas ideas, las acciones a las cuales se hace referencia constituyen parte de los procesos y una de ellas, la depuración es la encargada de encontrar errores en todos los procesos anteriores y corregirlos. De ahí que este proceso sea diferente en cada proyecto que responda a las necesidades de informatización de una organización. En proyectos de producción de sistemas, la depuración transcurre como se ha abordado en los párrafos anteriores, sin embargo, en otros proyectos no necesariamente tiene por qué ser así.

Existen proyectos de informatización en los cuales se desarrolla un sistema como parte del proceso, sin embargo, este sistema es solamente una acción dentro de otras acciones. En este caso, al ejecutarse el sistema de acciones modeladas, no se codifica en su totalidad, sino que es una parte de este; cuestión esta que modifica sustancialmente las concepciones de depuración ya analizadas. Otro caso es cuando no se realiza ninguna acción de codificación, pero se llevan a la práctica diferentes modelos obtenidos durante la marcha del proceso. Un ejemplo que ya ha sido tratado en este artículo es el de VoIP. En este caso se modela la arquitectura de la red a partir de las características de la pizarra telefónica y el conjunto de elementos a conectar en dependencia de los requisitos obtenidos.

A partir de la obtención del modelo se comienza la implantación de la red configurada según los modelos y consecuentemente se hacen pruebas muy parecidas que en el caso. Se comienza a realizar las pruebas de cada una de las acciones integrantes del proceso de informatización y se depurarán en dependencia de las características de cada tipo de proyecto. Para proyectos de redes las pruebas deben ser muy parecidas a las pruebas de las aplicaciones web en cuanto a su concepción. Quiere ello decir que debe probarse los sistemas informáticos involucrados, la infraestructura que los sustenta y las políticas implantadas. Una vez detectados los errores la corrección de los 
mismos puede pasar por la sustitución de un equipo hasta la instalación de nuevos sistemas. Es opinión del autor, que en cada uno de los casos analizados se mantienen las etapas genéricas de desarrollo de un software por lo cual se puede generalizar a los procesos de informatización en organizaciones. De ahí la importancia de la preparación de un profesional para la depuración de estos errores.

Esta afirmación en el párrafo anterior permite entonces considerar que la depuración es una de las formas de actuación del profesional informático con una gran repercusión en sus actividades profesionales. Se infiere entonces que los profesionales informáticos crean estrategias de pruebas para detectar errores y depurarlos como parte de su actividad para la informatización de los diferentes procesos organizacionales.

Al comprender la depuración como parte del modelo del profesional de la informática y una de las actividades más importantes como futuro informático en organizaciones, es necesario dilucidar si es una habilidad o una competencia. Para algunos autores reflejados en (Díaz, 2015) la modelación es una habilidad, pero no se refiere a la depuración. Esta conceptualización de la modelación como habilidad adolece de una insuficiencia que, a juicio de este autor, la lleva a restringir su estructura para el contexto de este artículo por lo tanto la depuración como proceso siguiente también sería una habilidad. La insuficiencia de estos autores radica fundamentalmente en la contextualización de la modelación en un currículo de formación básica que posee diferencias sustanciales respecto al currículo de formación profesional en el área de informática.

Para otros autores (Martínez-Artero y Nortes Checa, 2016) se estructuran habilidades generalizadas que tienen como característica esencial las acciones y operaciones generalizadas con un conocimiento que también es general. En esta definición percibe la depuración asociada a procesos de desarrollo de sistemas dejando de lado la depuración de procesos de informatización y aunque como aspecto positivo esta se concibe como parte del proceso de desarrollo de un sistema.

El profesional del área de informática debe incorporar la depuración en su actuación como uno de los requisitos esenciales de concreción de su actividad. En esta proyección y planificación de su actividad como futuro profesional se desprenden un conjunto de cualidades esenciales como la toma de decisiones en la proyección de procesos de informatización, la honestidad y responsabilidad al asumir los errores propios y del colectivo en la depuración de sistemas, entre otras. Ello implica que la depuración rebase a la habilidad como estructura sistémica.

Para varios autores (Sospedra y Rosa, 2015) las competencias pueden ser definidas como aprendizajes o logros complejos que integran aspectos cognitivos, procedimentales, actitudinales, habilidades, características de la personalidad y valores, que, puestos en práctica en un determinado contexto, tendrán un impacto positivo en los resultados de la actividad desempeñada.

La necesaria diferenciación de los procesos de depuración que cada estudiante realiza y que se estructura de manera individual posibilita la expresión de sus experiencias, conocimientos y habilidades relacionadas con este proceso de llevar a vías de hecho los modelos realizados. Ello les permite integrar sus proyectos con el resto e ir aprendiendo de los demás colegas. Teniendo en cuenta estos elementos es que se aprecia en este artículo que la depuración es una competencia en el ingeniero informático y a continuación se analizará su estructura. 
A continuación, se argumentará que la depuración es una competencia del ingeniero informático obviando el sistema de conocimientos que ya ha sido abordado en el primer acápite de este artículo, siendo necesario abordar las habilidades dentro de esta competencia. Se pueden enunciar cinco habilidades esenciales de un profesional informático relacionada con la depuración. Una primera se denomina en este artículo elaborar plan de pruebas que se representan el sistema de pruebas que se van a realizar utilizando el sistema de artefactos adecuados que le permite estructurar las pruebas que se han analizado hasta el momento. La segunda se denomina elaborar la estrategia de pruebas en los procesos de informatización y llevarlos a cabo según la concepción de los encargados de estos procesos: gestor de pruebas y probador. Para ello es importante retomar el proyecto, ahora como eje articulador de los procesos formativos en los ingenieros informáticos. La tercera habilidad está relacionada con la ejecución de las pruebas para la concreción del plan y la estrategia de pruebas en la solución de la problemática planteada al proyecto. Dentro de esta tercera habilidad, la selección de las herramientas automáticas, en caso que el proyecto los necesite, es importante para la depuración de los sistemas por variadas causas establecidas en la literatura (Black y Mitchell, 2011; Lacerda, 2007; Marques de Lima, Ribeiro de Lima, Coêlho Monteiro, Cavalcante Júnior, y Leal Gomes, 2012; Pressman, 2010). La cuarta habilidad está relacionada con la selección del método de depuración de estos procesos en la práctica. La última, y no menos importante, es la ejecución de la depuración de los errores detectados en el proceso de informatización de las organizaciones. La integración de estas cinco habilidades en un sistema armónico conjuntamente con los conocimientos acerca de la depuración permitirá a los estudiantes conformar con éxito un proyecto.

La formación basada en proyectos permite al estudiante aplicar los contenidos apropiados y analizar rápidamente la pertinencia de éstos para su formación profesional. En un entorno de proyecto el estudiante desempeña los roles de su futuro profesional y va articulando los modos de actuación que desempeñará en el futuro, de tal manera que se va articulando dentro de su proyecto de vida la futura profesión que va a desempeñar. Este proceso tiene una especial relevancia en la juventud por ser una de las características fundamentales de la situación social de desarrollo en la cual se encuentran.

Sin embargo, la educación de los valores en el proyecto es también importante a tener en cuenta en este artículo. En este mismo orden de ideas, se van conformando aquellos valores propios de la profesión como la responsabilidad, comprometido, honesto y humilde (Méndez, Ortega, y Lara, 2016). La responsabilidad es uno de los valores más importantes en el profesional informático por las características propias de la actividad, así como por el impacto social que tienen las tecnologías para la sociedad. En cualquiera de los roles que deba desempeñar debe ser responsable de sus actos y constituir un verdadero eje regulador de sus acciones puesto que de ellas depende las del resto del proyecto. La depuración es una de las actividades con potencial para el desarrollo de la responsabilidad por el papel que desempeña en la concreción del proceso de informatización a desarrollar y por su marcado carácter práctico. Además, es esencial el proceso de depuración para que el proceso de informatización carezca de errores y sea eficiente.

Durante el desarrollo del proyecto la honestidad con los colegas en el proceso de depuración es primordial para entender las relaciones sociales que se establecen. Estas relaciones en las cuales el proyecto, el posicionamiento de la empresa y la confianza entre los integrantes juegan un papel fundamental.

La educación de estos valores debe basarse en el conjunto de vivencias que hacen comprender al sujeto su responsabilidad ante los demás integrantes del proyecto y la 
sociedad por el resultado del proyecto que van a obtener. Este proceso debe estar centrado en la importancia del rol que desempeña y el resultado de la actividad para la organización. En un ambiente de proyecto todos los roles son importantes. Juega un papel esencial la explicación detallada de los errores, así como la oportunidad de expresarlos y corregirlos sin constituir una oportunidad de castigo, a través del diálogo, la confrontación y la polémica constante y constructiva. También es importante para la depuración el ir asignando tareas de mayor complejidad y que involucren con el resto de los integrantes del proyecto para explicar las especificidades de su proyecto en la organización.

La integración de estos valores, en las configuraciones que se estructuran en la actividad informática, con el proyecto de vida hace que se incorporen de manera real al potencial regulador de la personalidad del profesional. De esta manera estos valores se constituirán en parte de la subjetividad del sujeto y no generarán formalismos.

De la misma manera que los valores, se tienen en cuenta los restantes componentes de la competencia depuración de modelos. La integración de estudiante - realidad enseñanza propicia que el trabajo de los estudiantes adquiera un carácter social tanto por la implicación de los resultados del proyecto para las organizaciones, así como el sistema de relaciones a desarrollar con el resto del colectivo en la solución de los problemas. Lo anteriormente planteado conlleva al análisis de la situación y una postura reflexiva ante las críticas y los cuestionamientos.

La transformación de la realidad por parte del estudiante a partir del proceso de informatización y la selección de las herramientas necesarias para lograrlo evidencian el carácter activo de la función reguladora de su personalidad. La amplia variedad de herramientas para una misma actividad, así como la selección de las metodologías implica que se han tomado decisiones con respecto al proyecto y los modelos que en él intervienen. Este proceso de transformación debe ocurrir primeramente en el plano mental jugando un papel importante de la imaginación, con lo que se contribuye a su desarrollo. Este es uno de los aspectos esenciales que diferencian la depuración de la modelación. En este caso, por la posición que ocupa este proceso en el desarrollo del sistema, los estudiantes aprecian con mayor claridad cómo se da el tránsito de lo abstracto a lo concreto durante la actividad informática. De esta manera los estudiantes van integrando estas vivencias de participación en actividades de generación casos de pruebas, estrategias de pruebas entre otras actividades al mismo tiempo que van regulando el aprendizaje de la depuración en la actividad informática.

A partir de la búsqueda de problemas en la realidad se comienza el ciclo de vida de un software hasta que concluye con la puesta punto y mantenimiento. Los problemas derivados del proyecto individual motivan a los estudiantes hacia su solución y en ellos se encuentran los conocimientos del curso que a su vez generan las situaciones polémicas para los demás estudiantes. Las acciones y operaciones asociadas a la depuración, a partir de la utilización del conjunto de símbolos y signos asociados a ello, y el trabajo conjunto con el resto de colegas de mayor experiencia van estructurando diferentes escenarios de su futuro perfil profesional.

En cada encuentro, a partir de la interacción previa entre estudiante - profesor - grupo, se determinan las situaciones polémicas para los restantes estudiantes. Es el profesor quien decide la situación polémica a presentarse en el encuentro basándose previamente en la interacción grupal y el desarrollo de los proyectos de los estudiantes. La toma en cuenta de las situaciones polémicas asociadas a la depuración, y su solución, desde un proyecto les posibilita que se tengan en cuenta sus metas, proyectos y expectativas. En la medida que estas situaciones polémicas se estructuren y se 
concatenen con su práctica los lleva a reflexionar sobre esta práctica y mejorarla. En este mejoramiento continuo se va creciendo como profesional, propicia que se continúe el aprendizaje de sí mismo y de su aprendizaje de manera que propicie su regulación.

Para muchos autores (Gómez Gómez, 2013; Gutiérrez Alea, 2012; Martínez-Artero y Nortes Checa, 2016; Méndez et al., 2016; Sánchez, 2009) la formación de habilidades en la informática se da en el momento de enseñar un sistema en particular. En este artículo se asume una concepción sistémica propuesta por varios autores (González Hernández, 2013a; Mosquera, 2011). En esta concepción de la enseñanza de la informática se aborda el proceso como un todo integrado, en el cual se le presta especial atención a la concatenación de los conceptos y procedimientos informáticos que no se pueden formar en una única clase como en el caso que ocupa este artículo: la competencia depurar procesos de informatización a organizaciones. Por el carácter interdisciplinar y la complejidad de su estructura, su formación en el profesional informático debe abordarse desde una postura sistémica.

Aplicar el enfoque de sistema conlleva al análisis de los sistemas de aplicación porque anteceden a la enseñanza de la programación en la preparación informática de los estudiantes. Una de las cuestiones en la cual realiza énfasis la enseñanza de la Informática, por la importancia que reviste para esta ciencia, es el procesamiento de la información. La depuración de los procesos de informatización y sus flujos puede estructurarse desde los inicios de la formación informática y en las diferentes disciplinas informáticas que componen la formación del profesional de esta ciencia.

Para comprender la posición en este artículo sobre el desarrollo de la competencia implementar procesos de informatización es importante asumir diferentes posiciones. Existen hoy dos criterios divergentes en cuanto la formación informática y las disciplinas de programación e ingeniería de software. Algunos plantean que es preferible comenzar por la enseñanza de la programación y otros por la ingeniería del software. Cuando la enseñanza de la informática se estructura sobre la base del proyecto como eje formativo la ingeniería de software alcanza prevalencia pues es la proyección de lo que se quiere alcanzar. Es la disciplina que provee de los símbolos y sus relaciones que permiten lograr las primeras 4 habilidades necesarias para la comprensión de la representación del proceso a informatizar. Se forma entonces las habilidades representar procesos y estructuras y comprender representaciones que fueron definidas anteriormente. De esta manera se prepara al estudiante desde el aprendizaje para la futura actividad profesional que va a realizar. Estos procesos son necesarios que el estudiante los conozca antes de realizar un proceso de depuración en la concepción amplia sustentada en este artículo.

Asumiendo que la Ingeniería de Software deba anteceder a la programación es importante destacar que las Bases de Datos deben comenzar a enseñarse desde la introducción de la Ingeniería de Software para determinar el modelo entidad relación desde el dominio de la aplicación. De esta manera el estudiante se apropia de las formas de trabajo propias de cada asignatura, pero tributando a la modelación y a la depuración y a la estrecha relación que existe entre estas disciplinas informáticas. La integración de estas dos asignaturas en el proyecto le provee al estudiante de los elementos necesarios para realizar una primera modelación del análisis de la aplicación que le resultará necesario para comprender este proceso e implementarlo.

Posteriormente, al refinar este primer modelo de análisis se puede comenzar a enseñar programación orientada a objetos desde los inicios. El paradigma imperante es la programación orientada a objetos y se puede comenzar a analizar los conceptos de clase, objeto, herencia y polimorfismo. Posteriormente se abordan los conceptos de 
algoritmo, variable y código a partir de estas clases y los métodos que en ellas se incluyen. Este proceder metodológico propicia la integración de conocimientos tal y como transcurre en un proceso de desarrollo de software. Siguiendo este orden de ideas, la evaluación final de las asignaturas debe ser integrada para evaluar precisamente los objetivos de cada una de las asignaturas y la integración de ellas para resolver un proyecto real.

Cuando se enseña programación entonces se comienza a trabajar desde la modelación del sistema informático usando cualquiera de los elementos estructurales de los dos grandes grupos de metodologías: pesadas y ágiles para posteriormente lograr estructurar coherentemente la depuración tal y como se concibe en este artículo.

En este proceso se va desarrollando la habilidad comprender representaciones. El estudiante va analizando las representaciones realizadas por otros, relaciona los símbolos expresados que posee de su modelo y los va concatenando para la representación en el plano mental del proceso o estructura a informatizar para posteriormente implementarlo.

Ya en este momento, el estudiante puede estructurar mejor su accionar en función de ejecutar las acciones contenidas en el modelo, como parte del proceso de depuración dentro de su esfera de actuación como profesional de la informática. Este análisis vuelve a situar al estudiante ocupando los roles de su futuro como profesional. Sin embargo, el tratamiento de la depuración no acaba con la enseñanza de la programación.

Para este artículo la competencia implementar procesos de informatización es una de las más sistémicas e integradoras de la actividad profesional informática. Constituye un eje central en dos roles de la actividad informática: gestor de pruebas, probador y gestor de proyecto. Además, juega un papel esencial como concreción de los modelos obtenidos para las acciones de informatización de procesos. Por ende, la formación de competencia depurar procesos de informatización comienza en el segundo año de la carrera y culmina cuando el estudiante expresa su proceso de investigación en forma de memoria escrita en el cual integra todos los modelos estudiados en la carrera para describir el proyecto y los implementa. Ya el proceso de desarrollo de esta competencia corresponde a su ámbito laboral a partir de las diversas problemáticas que este profesional debe resolver en el ámbito organizacional.

Para este artículo la depurar sistemas para los procesos de informatización es una de las más sistémicas e integradoras de la actividad profesional informática. Constituye un eje central en dos roles de la actividad informática: gestor de pruebas, probador y gestor de proyecto. Además, juega un papel esencial como concreción de los modelos obtenidos para las acciones de informatización de procesos. Por ende, la formación de competencia depurar procesos de informatización comienza en el segundo año de la carrera y culmina cuando el estudiante expresa su proceso de investigación en forma de memoria escrita en el cual integra todos los modelos estudiados en la carrera para describir el proyecto y los implementa. Ya el proceso de desarrollo de esta competencia corresponde a su ámbito laboral a partir de las diversas problemáticas que este profesional debe resolver en el ámbito organizacional.

Por tanto se asume en este artículo que la competencia depurar sistemas es una configuración de recursos cognitivos, afectivos y autorreguladores relacionados con la depuración de sistemas que se adquieren durante la formación como profesional informático estrechamente ligado a la práctica en forma de proyectos, que como actividad transformadora de las organizaciones en las cuales se encuentran y se 
expresan a través de un desempeño, que en cumplimiento de los principios de la ética y el profesionalismo debe ser de calidad.

De esta definición se asume tres dimensiones que se declaran a continuación:

Cognitiva:

a) Amplio dominio de los conceptos asociados a las pruebas, errores y su depuración.

b) Elaborar plan de pruebas que se representan el sistema de pruebas que se van a realizar utilizando el sistema de artefactos adecuados que le permite estructurar las pruebas.

c) Elaborar la estrategia de pruebas en los procesos de informatización y llevarlos a cabo según la concepción de los encargados de estos procesos.

d) Ejecución de las pruebas para la concreción del plan y la estrategia de pruebas en la solución de la problemática planteada.

e) Selección del método de depuración de estos procesos en la práctica.

f) Elaboración de estrategias de depuración de los errores detectados durante el proceso de pruebas.

g) Ejecución de la depuración de los errores detectados en el proceso de informatización de las organizaciones.

Autorreguladores

a) Seleccionar el mejor plan de pruebas para la aplicación que se le va a aplicar.

b) Seleccionar los mejores tipos de pruebas en dependencia del tipo de aplicación a desarrollar.

c) Diseñar estrategias para corregir los errores que existan en el proceso de depuración.

d) Establecer mecanismos de retroalimentación en las acciones que implementa en el proceso de depuración

e) Establece metas para la ejecución de las acciones contenidas en la depuración de los sistemas.

f) Regula los procesos que han de ejecutarse para detectar la causa de los errores en el desarrollo de los sistemas.

Valores:

a) Asume con responsabilidad la ejecución de las pruebas que debe realizar para depurar los sistemas informáticos.

b) Asume con responsabilidad la búsqueda de las causas de los errores para su eliminación durante el proceso de depuración.

c) Propicia la creación de un clima de diálogo y polémica que estimule la crítica oportuna.

d) Institucionaliza el error como una oportunidad de aprendizaje para todo el equipo de depuración de los sistemas.

e) Exige el cumplimiento de los plazos acordados para la entrega en tiempo de los errores y su depuración.

f) Asumir la detección de errores como la función fundamental del tester en su relación social con el resto de los integrantes del equipo. 


\section{Métodos}

Participantes

Se toman como participantes a todos los 14 estudiantes de quinto año de ingeniería informática del curso 2016 - 2017 pues se cierra el ciclo de formación de la competencia con la depuración de las aplicaciones que desarrollarán en su proyecto de software. Durante el quinto año de la carrera los estudiantes deben finalizar un proyecto de software como un proceso de solución a una problemática de informatización en una empresa de software y se le denomina Trabajo de Diploma. En el segundo semestre del quinto año el departamento estructura un sistema de evaluaciones periódicas de la marcha de este proceso denominadas cortes en las cuales los estudiantes deben presentar el proyecto en diferentes estadios de desarrollo. Durante estas presentaciones se deben detectar los fallos del software y los estudiantes deben depurarlos para el próximo corte.

\section{Instrumentos}

Se aplicaron varios métodos de investigación

- Como parte de la encuesta se les entrega un cuestionario a estudiantes de quinto año: Se realizan 10 encuestas a los estudiantes del quinto año distribuidas en cuatro durante la asignatura de gestión de pruebas en el segundo semestre de cuarto año y en los 6 últimos cortes realizados. Esta distribución se realiza para contrastar los resultados obtenidos utilizando la triangulación de datos como se verá más adelante.

- Para realizar observaciones a los proyectos se aplica una guía de observación de los estudiantes: Se realizan 10 observaciones a proyectos de los estudiantes de la misma manera que se aplican las encuestas y durante el mismo período, cuestión esta que permitirá la triangulación de los datos al concluir todas.

- Las entrevistas a los profesores se apoyan en un cuestionario de preguntas: Se realizan entrevistas a los profesores al mismo tiempo que las encuestas a los estudiantes por las mismas razones ya expuestas.

- Técnica de ladov para medir satisfacción con el curso: esta técnica se utiliza para medir el nivel de satisfacción de los estudiantes con las acciones de pruebas al culminar su tesis.

\section{Procedimiento}

En la carrera de Ingeniería Informática de la Universidad de Matanzas los estudiantes reciben una asignatura denominada Gestión de Pruebas durante el cuarto año de la carrera. Esta asignatura es nueva en su currículo y comenzó a impartirse durante el curso 2015 - 2016 durante el primer semestre, por lo que se ha impartido durante dos cursos. Para la impartición de la asignatura se asume un enfoque de proyectos como se ha definido en la literatura al respecto (Giménez, 2016; González Hernández, 2013b; McGibbon y Belle, 2015; Morsy et al., 2015) y se les coloca como proyecto la depuración de los errores de los proyectos finales de los estudiantes de quinto año.

\section{Análisis de datos}

Se recogen los resultados de cada uno de los métodos aplicados para analizar la evolución de los estudiantes con presencia de los indicadores. Posteriormente se realizó la triangulación de los métodos empleados durante la aplicación práctica de la metodología con el objetivo de integrar los diferentes instrumentos que miden una 
misma dimensión, con similares aproximaciones en el mismo estudio para medir la variable. Al triangular la información obtenida se infieren las siguientes regularidades por las dimensiones establecidas en el proceso investigativo. Los resultados de los indicadores de estas dimensiones se encontraban en los métodos aplicados: encuesta a estudiantes, las observaciones a proyectos y la entrevista a a profesores.

\section{Resultados}

Con relación a las interrogantes número 1 y 2 del cuestionario de encuesta realizado a los estudiantes, donde se pretende que demuestren que poseen conocimientos sobre pruebas y las habilidades más importantes relacionadas con las pruebas se puede apreciar al inicio de la asignatura un total desconocimiento acerca de las pruebas y sus procesos más importantes. La selección se produce como resultado de una percepción errónea de las pruebas que han venido trabajando de varias metodologías anteriores. Como se puede apreciar, la cantidad de estudiantes que poseen desarrollo de la competencia se incrementa en el quinto año a partir de los proyectos reales que deben desarrollar.

En correspondencia a las interrogantes número 3 y 4 , referida a cuál es el criterio que ellos utilizan para la selección del plan de pruebas también existían muchos criterios erróneos sobre los elementos constituyentes del plan, así como el objetivo del mismo. Durante el curso los estudiantes van aprendiendo los elementos que lo componen y elaboran su propio plan de pruebas, aunque perfeccionan su implementación durante su proyecto de tesis y las prácticas laborales de los cuatro años.

En la pregunta 5 a los estudiantes si poseen vivencias relacionadas con las pruebas y cómo los profesores han influido en este proceso; ellos refieren que hasta el momento solamente habían analizado las pruebas como colocar datos para saber si la aplicación o los objetos devolvían los datos correctos. Ello evidencia que solamente se habían tratado las pruebas de unidad en las asignaturas anteriores. El número de estudiantes que incorporan nuevas vivencias relacionadas con las pruebas se incrementa durante el tiempo que dura la experiencia. Los resultados se pueden apreciar en la figura a continuación:

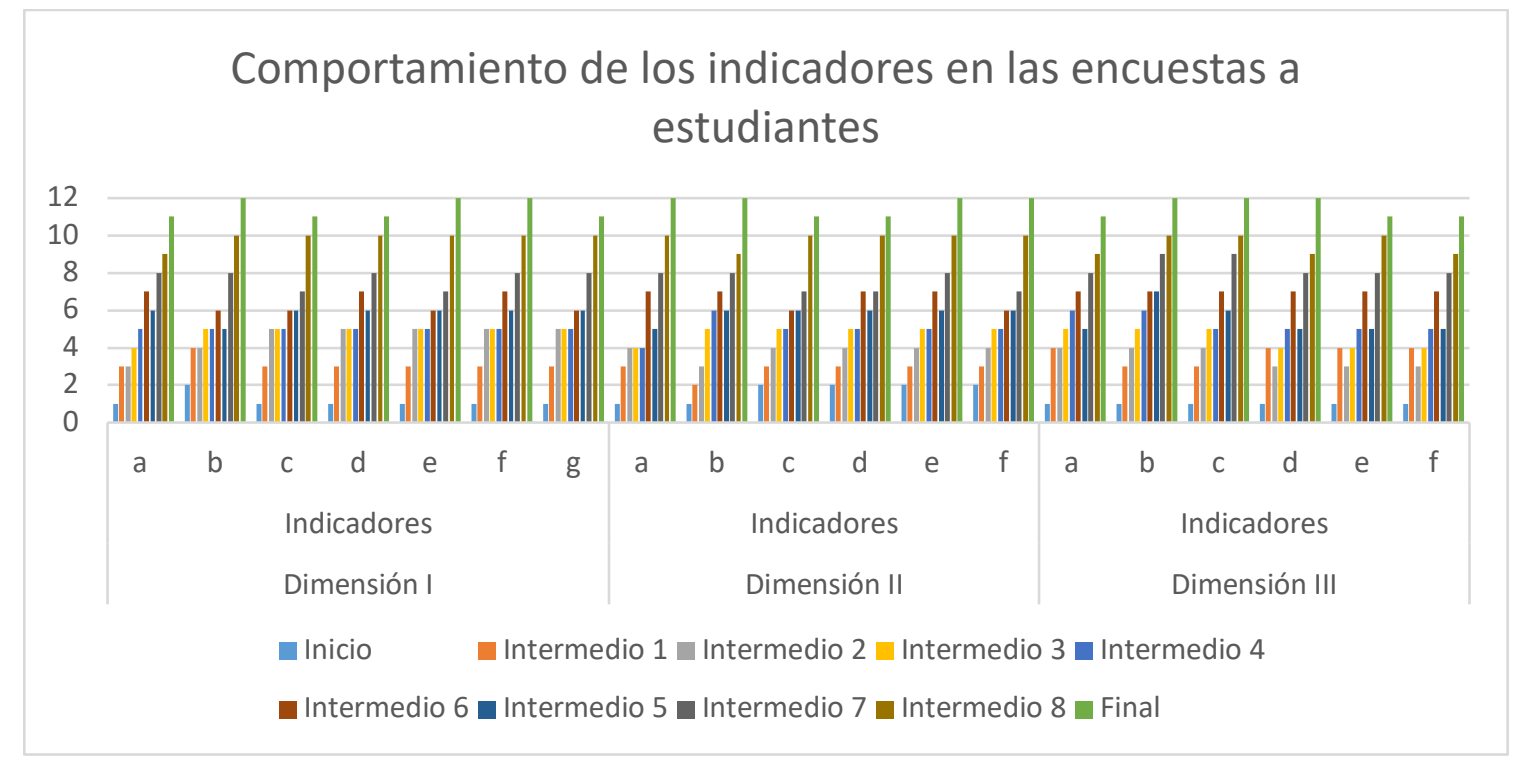

Figura \#1: Comportamiento de los indicadores de las tres dimensiones en las encuestas a los estudiantes. 
La práctica laboral se organizó según las necesidades de cada organización sobre la base de la utilización de una metodología de desarrollo y que el producto fuera una aplicación web. Dentro de la guía de la práctica laboral se les coloca la utilización de un modelo de pruebas. Durante los cortes los estudiantes conocen que el aspecto fundamental que será medido es el correcto funcionamiento de la aplicación y eso se garantiza estableciendo una correcta estrategia de depuración. En la ejecución de la observación se midieron los indicadores los indicadores de la variable dependiente que podían ser medibles con este instrumento. Durante la primera observación se constata un pobre desarrollo de los indicadores, solamente se constata por encima del $10 \%$ el indicador relacionado con la utilización del lenguaje simbólico. Los valores obtenidos en el resto de los indicadores permiten inferir que no existe responsabilidad ni cumplimiento de los plazos en los procesos iniciales. Los resultados de cada una de las observaciones de la práctica laboral demuestran un incremento gradual en los valores de cada indicador, aunque se constata que los avances no se producen de igual manera en todos. Los indicadores de incremento más lento se observan en aquellos relacionados con los procesos del futuro como tester y asumir esa función como un rol en su futuro desempeño. Los principales resultados se muestran en la gráfica a continuación:

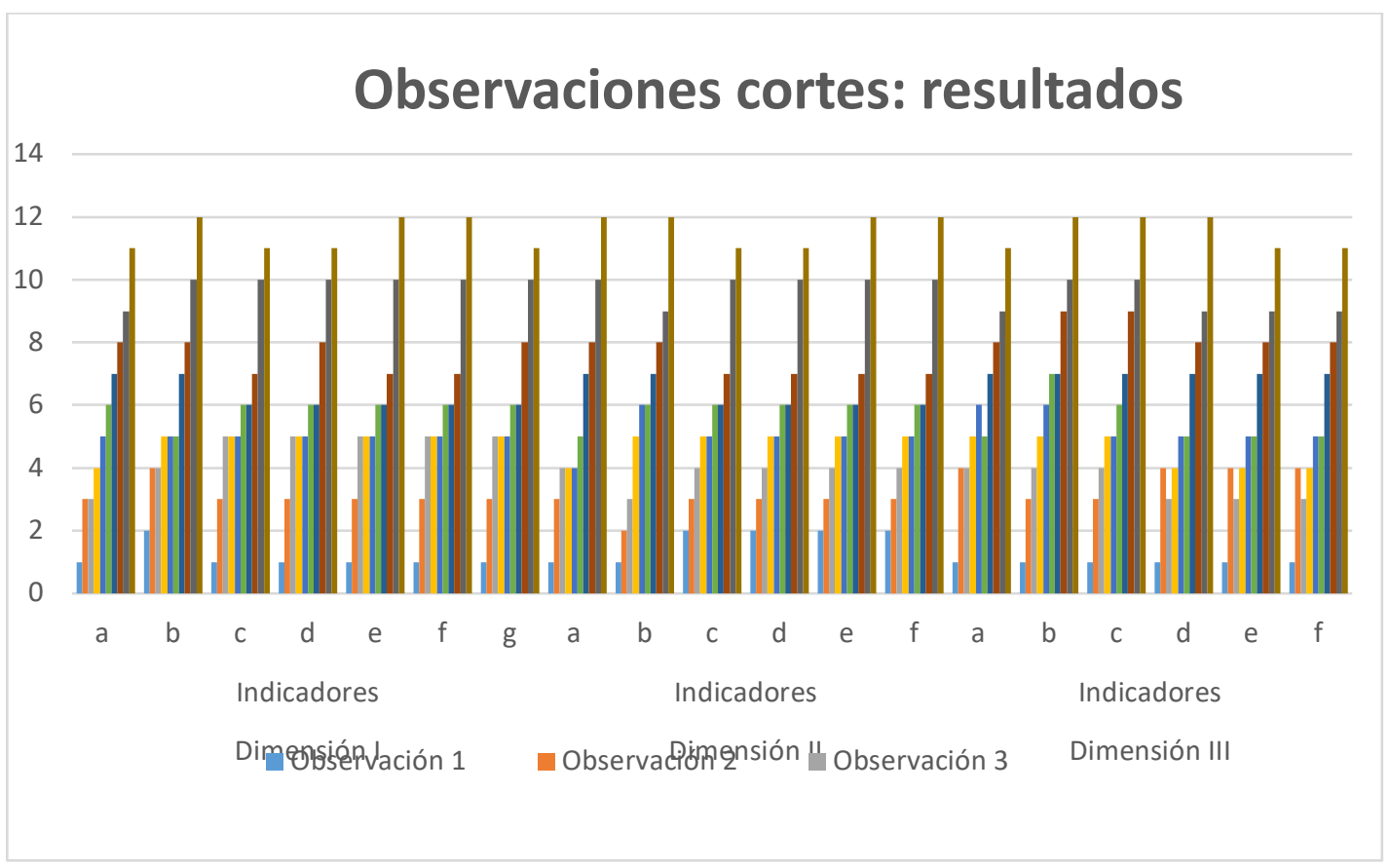

Figura \#2: Comportamiento de los indicadores de las tres dimensiones en las observaciones a los cortes de los estudiantes.

Las interrogantes de la entrevista a los profesores están relacionadas con las encuestas a los estudiantes pues se busca contrastar la opinión de los estudiantes con la de los profesores. Con relación a las interrogantes número 1 y 2 del cuestionario, donde se pretende que los estudiantes demuestran que poseen conocimientos sobre pruebas y las habilidades más importantes relacionadas con las pruebas los profesores concuerdan con los resultados de los estudiantes, aunque existen pequeñas variaciones en la cantidad de estudiantes, siempre menor en algunos indicadores en el análisis de los profesores. Para los profesores se ve un crecimiento en la cantidad de estudiantes que poseen desarrollo de la competencia durante el quinto año a partir de los proyectos reales que deben desarrollar.

De la misma manera que en las preguntas anteriores también se aprecia la existencia de muchos criterios erróneos sobre los elementos constituyentes del plan, así como el 
objetivo del mismo. Los profesores opinan que los estudiantes van aprendiendo los elementos que lo componen y elaboran su propio plan de pruebas, aunque perfeccionan su implementación durante su proyecto de tesis y las prácticas laborales de los cuatro años. Sus resultados se explicitan en la siguiente gráfica:

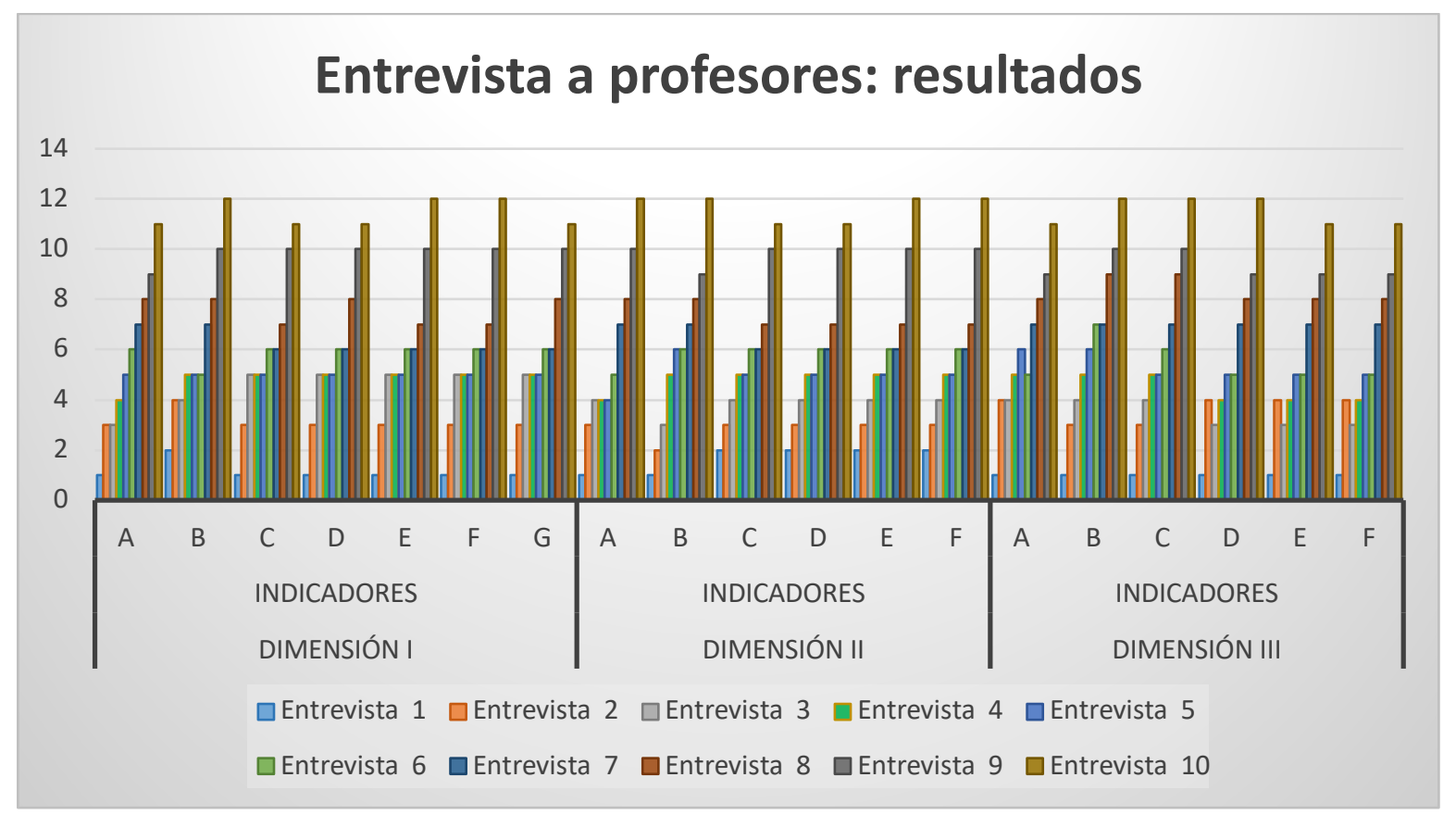

Figura \#3: Comportamiento de los indicadores de las tres dimensiones en las entrevistas a los profesores.

Se comienza entonces la triangulación de los datos de los indicadores que se encuentran en los tres métodos aplicados: entrevistas a profesores, encuestas a los estudiantes y observaciones a los cortes, en los dos primeros casos para contrastar entre la respuesta a la entrevista con la actuación del profesor y en las encuestas para constatar la evolución de los estudiantes. Como se puede apreciar en la gráfica la cantidad de estudiantes con resultados favorables en el desarrollo de estos indicadores fue aumentando en la medida que se fue aplicando las acciones del curso y del proyecto informático.

El incremento sostenido en la cantidad de estudiantes que fueron evaluados de positivo en estos indicadores puede observarse en las tablas correspondientes ya expresadas en esta tesis. Los resultados expresados en la gráfica a continuación llevan al autor a afirmar que la competencia depurar sistemas informáticos se forma en estos estudiantes. 


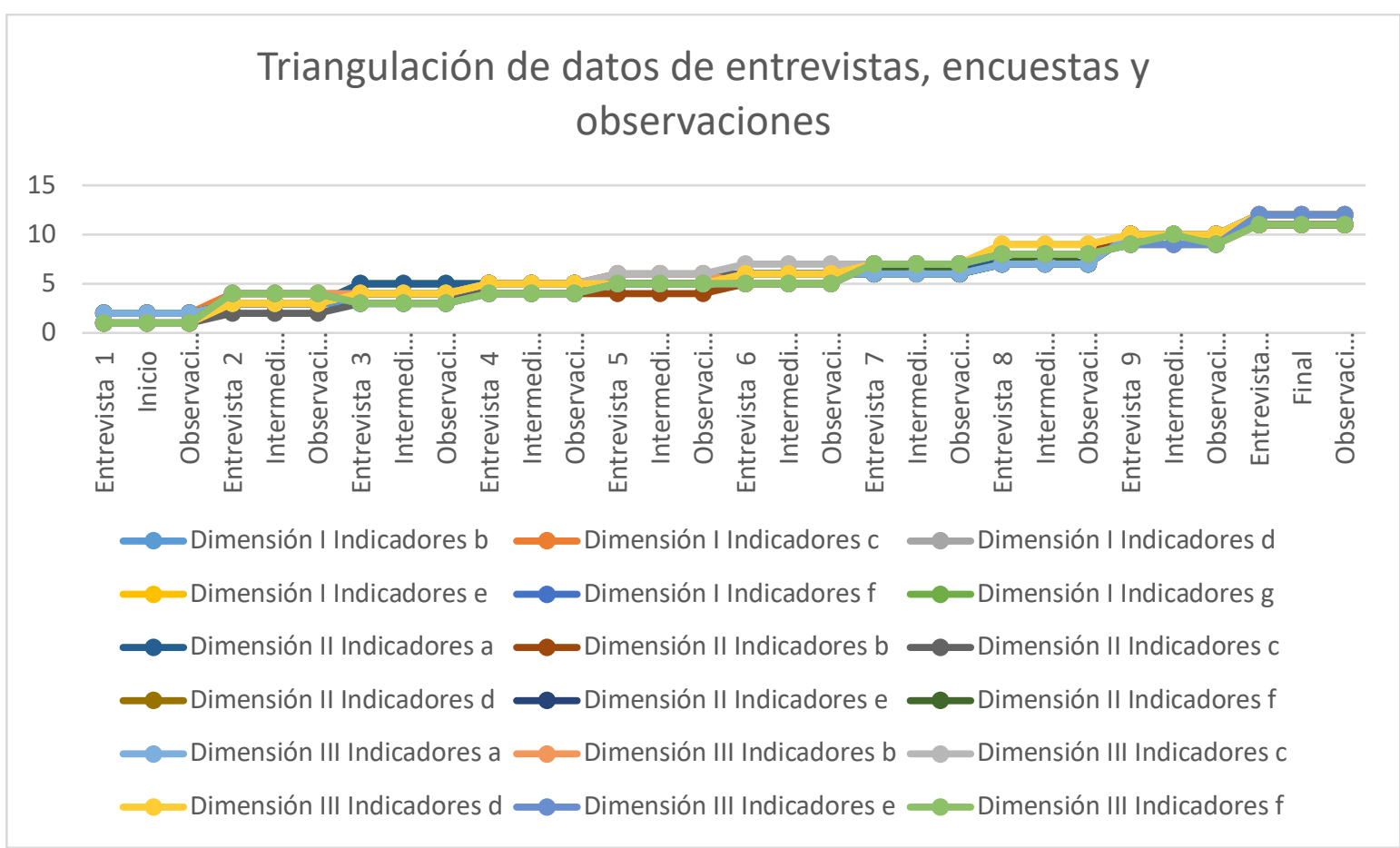

Figura \#4: Comportamiento de los indicadores de las tres dimensiones en triangulación a los datos obtenidos en los tres métodos.

\section{Discusión}

Del análisis de las tablas y gráficas expresadas anteriormente se pueden extraer las siguientes conclusiones:

Se aprecia que existe un incremento de la presencia de los indicadores lo que evidencia el impacto que tiene la vinculación de los estudiantes a proyectos reales en el desarrollo de la competencia depurar sistemas informáticos. Resulta interesante que las opiniones de los estudiantes y los profesores coinciden en pocos estudiantes con desarrollo de los indicadores. Se aprecia en los estudiantes un espíritu crítico que les permite el análisis de sus deficiencias, así como trazar estrategias de corrección.

La contrastación de métodos en el análisis de las tres dimensiones permite afirmar que la cantidad de estudiantes detectados con presencia de estos indicadores presentan resultados muy cercanos entre ellos a los obtenidos de la entrevista a los profesores. Ello permite afirmar que la triangulación de los métodos permite un mayor nivel de veracidad en cuanto la cantidad de estudiantes con desarrollo de la depuración como competencia.

Un elemento interesante que se resalta en la triangulación de los métodos está en el aumento de la cantidad de estudiantes con presencia de estos indicadores después de la práctica laboral. De estos resultados se corrobora la tesis expresada en la literatura al respecto (González, 2013) que el asumir el proyecto como eje en la formación de los profesionales informáticos potencia el desarrollo de su creatividad.

Además, se infiere que existen estudiantes con presencia de los indicadores de manera aislada que no se logran integrar en una configuración al no poseer los ejes centrales aglutinadores detectados en la experiencia práctica: 


\section{La incorporación de la actividad informática como tendencia orientadora de su personalidad.}

\section{Dominio de la informática como ciencia y su aplicación a las más diversas esferas de su actuación en determinadas actividades.}

\section{Autoconocimiento de sí mismo que le permite estructurar estrategias de aprendizaje regulando su actuación durante su proceso de formación.}

\section{Referencias}

Black, R., y Mitchell, J. L. (2011). Advanced Software Testing. Guide to the ISTQB Advanced Certification as an Advanced Technical Test Analyst. USA: O'Reilly Media.

Díaz, A. M. (2015). Evaluación de la propuesta de enseñanza de las áreas de lenguaje y matemáticas en la institución educativa san José del pantano. Panorama, 9(16), 25 - 39.

Giménez, D. (2016). Un curso práctico de Programación Paralela basado en problemas de Concurso Español de Programación Paralela. Paper presented at the XXII Jornadas sobre la Enseñanza Universitaria de la Informática, Almería, España.

Gómez Gómez, J. C. (2013). Caracterización de las prácticas evaluativas de los docentes de matemática de la institución educativa los palmitos, sucre - Colombia. Escenarios, 13, 96 - 107.

González Hernández, W. (2013a). Creativity Development in Informatics Teaching Using the Project Focus. International Journal of Engineering Pedagogy (iJEP), 3(1), 63-70.

González Hernández, W. (2013b). Creativity Development in Informatics Teaching Using the Project Focus. IJEP, 3(1), 22 - 30.

Gutiérrez Alea, M. (2012). Una metodología para contribuir al desarrollo de la habilidad resolver problemas en la disciplina Lenguajes y Técnicas de Programación, en estudiantes de la carrera de Licenciatura en Educación, especialidad de Informática. (Doctor en Ciencias Pedagógicas), Universidad Pedagógica Enrique José Varona, Academia de Ciencias de Cuba.

Lacerda, R. d. A. (2007). Proposta de um modelo para análise de requisitos de software educativo. (Programa de Pós-Graduação em Educação - PPGE), Universidade de Brasília - UnB, Brasília.

Marques de Lima, M., Ribeiro de Lima, A., Coêlho Monteiro, A. C., Cavalcante Júnior, E. H., y Leal Gomes, L. d. Q. (2012). Uma Revisão Sistemática da Literatura dos Processos de Desenvolvimento de Software Educativo. Paper presented at the Simpósio Brasileiro de Informática na Educação Rio de Janeiro.

Martínez-Artero, R. N., y Nortes Checa, A. (2016). La evaluación en Matemáticas en el Grado de Maestro de Primaria. Números. revista de Didáctica de las Matemáticas, 92, 57 - 70.

McGibbon, C., y Belle, J.-P. V. (2015). Integrating environmental sustainability issues into the curriculum through problem-based and project-based learning: a case study at the University of Cape Town. Current Opinion in Environmental Sustainability, 16, 81 - 88

Méndez, E. R. L., Ortega, A. M. G., y Lara, M. C. (2016). Modelo didáctico que contribuya a la interdisciplinariedad en el proceso de enseñanza-aprendizaje de la formación inicial de los profesores de Ciencias Naturales. Órbita Científica, 22(88).
Morsy, M. H., Alsareii, S. A., Al-Qahtani, J. M., Alshiek4, M. H. AlAyed2, M. S., y Abdullah5, M. (2015). Credit Hours Policy - Is It Working for Hybrid Problem-Based Learning Curriculum: An Experience of Najran School of Medicine KSA. Journal of Research in Medical Education y Ethics, 5(2), 129-133.

Mosquera, O. (2011). El reconocimiento del concepto función en estudiantes de la carrera de Ingeniería Industrial. Matanzas. (Tesis en opción al título de Master en Matemática Educativa), Universidad de Matanzas "Camilo Cienfuegos", Matanzas.

Pressman, R. S. (2010). Software engineering: A practitioner's approach. (Seventh Edition ed.). New York: McGraw-Hill.

Sánchez, B. I. (2009). El concepto de función matemática entre los docentes a través de representaciones sociales. (Tesis en opción al grado científico de Doctor en Matemática), Instituto Politécnico Nacional, México, D.F.

Sospedra, M., y Rosa, D. (2015). La formación docente universitaria en Cuba: Sus fundamentos desde una perspectiva desarrolladora del aprendizaje y la enseñanza. Estudios pedagógicos (Valdivia), $41(1), 337-349$. 